\title{
Operational and Variational Formulations of Boundary Problems of Anisotropic Plate Analysis, Adapted for Application Within Discrete-Continual Methods
}

\author{
Pavel Akimov ${ }^{1,2, *}$, Vladimir Sidorov ${ }^{3,4,5}$, Zbigniew Wójcicki ${ }^{6}$ \\ ${ }^{1}$ Russian Academy of Architecture and Construction Sciences; 24-1, ul. Bolshaya Dmitrovka, \\ Moscow, 107031, Russia \\ ${ }^{2}$ Scientific Research Center "StaDyO"; office 810, ul. 3ya Yamskogo Polya, Moscow, 125040, Russia \\ ${ }^{3}$ Kielce University of Technology; Al. 1000-lecia Państwa Polskiego 5, 25-541, Kielce, Poland \\ ${ }^{4}$ Moscow State University of Railway Engineering; 9b9 Obrazcova Street, Moscow, 127994, Russia \\ ${ }^{5}$ Perm National Research Polytechnic University; 29 Komsomolsky prospekt, Perm, Perm Krai, \\ 614990, Russia \\ ${ }^{6}$ Civil Engineering Faculty, Wrocław University of Technology, Wyb. Wyspiańskiego 27, 50-370 \\ Wrocław, Poland
}

\begin{abstract}
This paper is devoted to operational and variational formulations [1] of boundary problems of anisotropic plate analysis (with the use of so-called method of extended domain [2]), adapted for applications within discrete-continual methods (discrete-continual finite element method (DCFEM), discrete-continual variation-difference method (DCVDM) ) [1]. Generally, the field of application of these methods, which are now becoming available for computer realization, comprises structures with regular (in particular, constant or piecewise constant) physical and geometrical parameters in some dimension. Considering problems remain continual along "basic" direction while along other directions discretecontinual methods presuppose mesh approximation.
\end{abstract}

\section{Introduction}

As is known, structures from anisotropic materials are widely used in modern construction. Anisotropic materials normally have sharp difference in the elastic properties for different directions. A classic vital sample here is natural wood. Its elastic modulus in tension parallel to the grain is well above corresponding modulus in tension across the grain. Besides, elastic constants of natural wood depend on the direction with respect to the wood grains. Anisotropic (and inhomogeneous) materials are also include composite materials (particularly rather popular in aircraft engineering), crystals, some rocks, concrete, etc. Here we indicated materials with "natural" anisotropy. However, materials with so-called "artificial" anisotropy are applied in construction as well. We should mention here as a samples corrugated plates and shells from isotropic material or rib-

* Corresponding author: pavel.akimov@gmail.com 
reinforced plates and shell. Thus we have to consider boundary problems of anisotropic plate analysis [3-6].

\section{Standard operational and variational formulations of boundary problem within method of extended domain}

Operational formulation of the problem in the domain $\omega$ has the form:

$$
\begin{aligned}
\sum_{i=1}^{2} \sum_{j=1}^{2} \partial_{j}^{2} \bar{B}_{i, j} \partial_{i}^{2} w+4 \partial_{1} \partial_{2} \bar{B}_{3,3} \partial_{1} \partial_{2} w+ \\
\quad+2 \sum_{i=1}^{2}\left(\partial_{i}^{2} \bar{B}_{i, 3} \partial_{1} \partial_{2} w_{k}+\partial_{1} \partial_{2} \bar{B}_{i, 3} \partial_{i}^{2} w_{k}\right)+\bar{c} w=\theta q+\delta_{\Gamma}^{\prime} M_{\Gamma}+\delta_{\Gamma} Q_{\Gamma},
\end{aligned}
$$

where $\Omega$ is the domain, occupied by structure with boundary $\Gamma=\partial \Omega ; \omega$ is extended domain, embordering $\Omega ; x_{1}, x_{2}$ are coordinates in plan; $x_{3}$ is coordinate of plate thickness; $\theta=\theta\left(x_{1}, x_{2}\right)$ is the characteristic function of domain $\Omega ; \delta_{\Gamma}=\delta_{\Gamma}\left(x_{1}, x_{2}\right)$ is the delta-function of border $\Gamma=\partial \Omega ; \bar{n}=\left[\begin{array}{ll}n_{1} & n_{2}\end{array}\right]^{T}$ is unit normal vector of domain boundary $\Gamma=\partial \Omega ; \delta_{\Gamma}^{\prime}=\delta_{\Gamma}^{\prime}\left(x_{1}, x_{2}\right)$ is corresponding normal derivative; $w$ is plate deflection in domain $\Omega ; q$ is the load density in domain $\Omega ; Q_{\Gamma}, M_{\Gamma}$ are shear force and torque moment at the border $\Gamma=\partial \Omega ; c$ is modulus of foundation;

$$
\begin{gathered}
\partial_{k}=\partial / \partial x_{k}, \partial_{k}^{*}=-\partial / \partial x_{k}, k=1,2 ; \\
\bar{c}=\theta c ; \quad \delta_{\Gamma}^{\prime}=\delta_{\Gamma}^{\prime}\left(x_{1}, x_{2}\right)=\partial \delta_{\Gamma}\left(x_{1}, x_{2}\right) / \partial \bar{n} ;
\end{gathered}
$$

$B_{1,1}, B_{2,2}, B_{3,3}, B_{1,2}=B_{2,1}, B_{2,3}=B_{3,2}, B_{1,3}=B_{3,1}$ are constants (parameters) that characterize the elastic properties of structure;

$$
\begin{aligned}
\bar{B}_{1,1}=\theta B_{1,1}, \bar{B}_{1,3}=\theta B_{1,3}, \bar{B}_{2,3}=\theta B_{2,3}, \bar{B}_{1,2} & =\theta B_{1,2}, \bar{B}_{3,3}=\theta B_{3,3} ; \\
& \bar{B}_{1,2}=\theta B_{1,2}, \bar{B}_{3,3}=\theta B_{3,3}, \bar{B}_{2,2}=\theta B_{2,2} ;
\end{aligned}
$$

and in the particular case of an isotropic plate, we have

$$
B_{1,1}=B_{2,2}=D ; \quad B_{1,2}=B_{2,1}=D v ; \quad B_{3,3}=0.5 \cdot D(1-v) ; \quad B_{1,3}=B_{2,3}=B_{3,1}=B_{3,2}=0 .
$$

Internal moments and plate forces are determined by formulas

$$
\begin{gathered}
M_{1}=B_{1,1} \chi_{1}+B_{1,2} \chi_{2}+B_{1,3} \chi_{12} ; \quad M_{2}=B_{1,2} \chi_{1}+B_{2,2} \chi_{2}+B_{2,3} \chi_{12} ; \\
M_{12}=M_{21}=B_{1,3} \chi_{1}+B_{2,3} \chi_{2}+B_{3,3} \chi_{12} ; \\
N_{1}=B_{1,1} \partial_{1} \chi_{1}+3 B_{1,3} \partial_{2} \chi_{1}+\left(B_{1,2}+2 B_{3,3}\right) \partial_{1} \chi_{2}+B_{2,3} \partial_{2} \chi_{2} ; \\
N_{2}=B_{1,3} \partial_{1} \chi_{1}+\left(B_{1,2}+2 B_{3,3}\right) \partial_{2} \chi_{1}+3 B_{2,3} \partial_{1} \chi_{2}+B_{2,2} \partial_{2} \chi_{2} ;
\end{gathered}
$$

where $\chi_{1}, \chi_{2}, \chi_{12}=\chi_{21}$ are strains for $x_{3}=1$,

$$
\chi_{1}=-\partial_{1}^{2} w ; \quad \chi_{2}=-\partial_{2}^{2} w ; \quad \chi_{12}=\chi_{21}=-2 \partial_{1} \partial_{2} w
$$


Variational formulation of the problem is formulated in the form of the corresponding energy functional (this formulation is convenient in the algorithmic sense):

$$
\Phi(w)=\frac{1}{2} \iint_{\omega}\left[\theta\left(M_{1} \chi_{1}+M_{2} \chi_{2}+2 M_{1,2} \chi_{1,2}\right)+\bar{c} w^{2}\right] d x_{1} d x_{2}-\iint_{\omega} q w d x_{1} d x_{2} .
$$

\section{Operational and variational displacement formulations of boundary problem within method of extended domain}

If we combine (6)-(10) we can get formulation (1) in the following form

$$
L w=F,
$$

where $L$ is so-called stiffness operator,

$$
\begin{gathered}
\mathrm{L}=\partial_{1}^{2} \bar{B}_{1,1} \partial_{1}^{2}+\partial_{2}^{2} \bar{B}_{1,2} \partial_{1}^{2}+\partial_{1}^{2} \bar{B}_{2,1} \partial_{2}^{2}+\partial_{2}^{2} \bar{B}_{2,2} \partial_{2}^{2}+4 \partial_{1} \partial_{2} \bar{B}_{3,3} \partial_{1} \partial_{2}+ \\
+2 \partial_{1}^{2} \bar{B}_{1,3} \partial_{1} \partial_{2}+2 \partial_{2}^{2} \bar{B}_{2,3} \partial_{1} \partial_{2}+2 \partial_{1} \partial_{2} \bar{B}_{1,3} \partial_{1}^{2}+2 \partial_{1} \partial_{2} \bar{B}_{2,3} \partial_{2}^{2}+\bar{c} \\
\mathrm{~F}=\theta q+\delta_{\Gamma}^{\prime} M_{\Gamma}+\delta_{\Gamma} Q .
\end{gathered}
$$

We can rewrite (11) in matrix form:

$$
\Phi(w)=\frac{1}{2} \iint_{\omega}\left[(\theta \bar{N}, \bar{\chi})+\bar{c} w^{2}\right] d x_{1} d x_{2}-\iint_{\omega} q w d x_{1} d x_{2},
$$

where

$$
\bar{N}=\left[\begin{array}{llll}
M_{1} & M_{2} & M_{1,2} & M_{2,1}
\end{array}\right]^{T} ; \quad \bar{\chi}=\left[\begin{array}{llll}
\chi_{1} & \chi_{2} & \chi_{1,2} & \chi_{2,1}
\end{array}\right]^{T} .
$$

Using (6)-(9), we get

$$
\bar{N}=A \bar{\chi}, \quad \bar{\chi}=B w,
$$

where

$$
A=\left[\begin{array}{llll}
B_{1,1} & B_{1,2} & 0.5 \cdot B_{1,3} & 0.5 \cdot B_{1,3} \\
B_{1,2} & B_{2,2} & 0.5 \cdot B_{2,3} & 0.5 \cdot B_{2,3} \\
B_{1,3} & B_{2,3} & 0.5 \cdot B_{3,3} & 0.5 \cdot B_{3,3} \\
B_{1,3} & B_{2,3} & 0.5 \cdot B_{3,3} & 0.5 \cdot B_{3,3}
\end{array}\right] ; \quad B=-\left[\begin{array}{c}
\partial_{1}^{2} \\
\partial_{2}^{2} \\
2 \partial_{1} \partial_{2} \\
2 \partial_{1} \partial_{2}
\end{array}\right]
$$

Thus we have:

$$
\Phi(w)=\frac{1}{2} \iint_{\omega}\left[\left(B^{*} \bar{A} B w, w\right)+\bar{c} w^{2}\right] d x_{1} d x_{2}-\iint_{\omega} q w d x_{1} d x_{2},
$$

where

$$
\bar{A}=\theta A=\left[\begin{array}{llll}
\bar{B}_{1,1} & \bar{B}_{1,2} & 0.5 \cdot \bar{B}_{1,3} & 0.5 \cdot \bar{B}_{1,3} \\
\bar{B}_{1,2} & \bar{B}_{2,2} & 0.5 \cdot \bar{B}_{2,3} & 0.5 \cdot \bar{B}_{2,3} \\
\bar{B}_{1,3} & \bar{B}_{2,3} & 0.5 \cdot \bar{B}_{3,3} & 0.5 \cdot \bar{B}_{3,3} \\
\bar{B}_{1,3} & \bar{B}_{2,3} & 0.5 \cdot \bar{B}_{3,3} & 0.5 \cdot \bar{B}_{3,3}
\end{array}\right] ; \quad B^{*}=-\left[\begin{array}{llll}
\partial_{1}^{2} & \partial_{2}^{2} & 2 \partial_{2} \partial_{1} & 2 \partial_{2} \partial_{1}
\end{array}\right]
$$




\section{Operational and variational formulations of boundary problem in displacements with extraction of basic direction}

Without loss of generality we suppose constancy of physical and geometrical parameters of plate along coordinate $x_{2}$ (it is so-called "basic direction").

We can rewrite (12) in the following form:

$$
L=-L_{4} \partial_{2}^{4}+L_{3} \partial_{2}^{3}+L_{2} \partial_{2}^{2}+L_{1} \partial_{2}+L_{0}+\bar{c},
$$

where

$$
\begin{aligned}
& L_{4}=-\bar{B}_{2,2} ; \quad L_{3}=2 \bar{B}_{2,3} \partial_{1}+2 \partial_{1} \bar{B}_{2,3} ; \quad L_{2}= \bar{B}_{1,2} \partial_{1}^{2}+\partial_{1}^{2} \bar{B}_{2,1}+4 \partial_{1} \bar{B}_{3,3} \partial_{1} ; \\
& L_{1}=2 \partial_{1}^{2} \bar{B}_{1,3} \partial_{1}+2 \partial_{1} \bar{B}_{1,3} \partial_{1}^{2} ; \quad L_{0}=\partial_{1}^{2} \bar{B}_{1,1} \partial_{1}^{2} .
\end{aligned}
$$

We can rewrite (21):

$$
-L_{4} \partial_{2}^{4} w+L_{3} \partial_{2}^{3} w+L_{2} \partial_{2}^{2} w+L_{1} \partial_{2} w+\left(L_{0}+\bar{c}\right) w=F .
$$

Let us introduce the following notation

$$
y_{1}=y_{1}\left(x_{1}, x_{2}\right)=w\left(x_{1}, x_{2}\right) ; \quad y_{i}=y_{i}\left(x_{1}, x_{2}\right)=\partial_{2}^{i-1} w\left(x_{1}, x_{2}\right), \quad i=2,3,4 .
$$

If we combine (23) and (24) we get:

$$
-L_{4} \partial_{2} y_{4}+L_{3} y_{4}+L_{2} y_{3}+L_{1} y_{2}+\left(L_{0}+\bar{c}\right) y_{1}=F .
$$

Uniting (24) and (25) we obtain

$$
\left[\begin{array}{llll}
1 & 0 & 0 & 0 \\
0 & 1 & 0 & 0 \\
0 & 0 & 1 & 0 \\
0 & 0 & 0 & L_{4}
\end{array}\right]\left[\begin{array}{l}
y_{1}^{\prime} \\
y_{2}^{\prime} \\
y_{3}^{\prime} \\
y_{4}^{\prime}
\end{array}\right]=\left[\begin{array}{cccc}
0 & 1 & 0 & 0 \\
0 & 0 & 1 & 0 \\
0 & 0 & 0 & 1 \\
L_{0}+\bar{c} & L_{1} & L_{2} & L_{3}
\end{array}\right]\left[\begin{array}{l}
y_{1} \\
y_{2} \\
y_{3} \\
y_{4}
\end{array}\right]-\left[\begin{array}{c}
0 \\
0 \\
0 \\
F
\end{array}\right],
$$

where

$$
y_{i}^{\prime}\left(x_{1}, x_{2}\right)=\partial_{2} y_{i}\left(x_{1}, x_{2}\right), \quad i=2,3,4 \text {. }
$$

Thus we have the following system of the first-order ordinary differential (with respect to $x_{2}$ ) equations with operator coefficients

$$
\left[\begin{array}{l}
y_{1}^{\prime} \\
y_{2}^{\prime} \\
y_{3}^{\prime} \\
y_{4}^{\prime}
\end{array}\right]=\left[\begin{array}{cccc}
0 & 1 & 0 & 0 \\
0 & 0 & 1 & 0 \\
0 & 0 & 0 & 1 \\
\mathrm{~L}_{4}^{-1}\left(\mathrm{~L}_{0}+\bar{c}\right) & \mathrm{L}_{4}^{-1} \mathrm{~L}_{1} & \mathrm{~L}_{4}^{-1} \mathrm{~L}_{2} & \mathrm{~L}_{4}^{-1} \mathrm{~L}_{3}
\end{array}\right]\left[\begin{array}{l}
y_{1} \\
y_{2} \\
y_{3} \\
y_{4}
\end{array}\right]-\left[\begin{array}{c}
0 \\
0 \\
0 \\
\mathrm{~L}_{4}^{-1} \mathrm{~F}
\end{array}\right]
$$

or

$$
\bar{U}^{\prime}=\widetilde{L} \bar{U}+\overline{\widetilde{F}}
$$

where 


$$
\begin{gathered}
\widetilde{L}=\left[\begin{array}{cccc}
0 & 1 & 0 & 0 \\
0 & 0 & 1 & 0 \\
0 & 0 & 0 & 1 \\
L_{4}^{-1}\left(L_{0}+\bar{c}\right) & L_{4}^{-1} L_{1} & L_{4}^{-1} L_{2} & L_{4}^{-1} L_{3}
\end{array}\right] ; \quad \overline{\widetilde{F}}=-\left[\begin{array}{c}
0 \\
0 \\
0 \\
L_{4}^{-1} F
\end{array}\right] ; \\
\bar{U}=\left[\begin{array}{l}
y_{1} \\
y_{2} \\
y_{3} \\
y_{4}
\end{array}\right] ; \quad \bar{U}^{\prime}=\partial_{2} \bar{U}=\left[\begin{array}{l}
\partial_{2} y_{1} \\
\partial_{2} y_{2} \\
\partial_{2} y_{3} \\
\partial_{2} y_{4}
\end{array}\right]=\left[\begin{array}{c}
y_{1}^{\prime} \\
y_{2}^{\prime} \\
y_{3}^{\prime} \\
y_{4}^{\prime}
\end{array}\right] .
\end{gathered}
$$

Equations (29), of course, should be supplemented with boundary conditions given in cross-sections with coordinates $x_{2, k}^{b}, k=1, \ldots, n_{k}$ This boundary conditions have form

$$
\begin{gathered}
B_{k}^{-} \bar{U}_{k-1}\left(x_{2, k}^{b}-0\right)+B_{k}^{+} \bar{U}_{k}\left(x_{2, k}^{b}+0\right)=\bar{g}_{k}^{-}+\bar{g}_{k}^{+}, \quad k=2, \ldots, n_{k}-1 ; \\
B_{1}^{+} \bar{U}_{1}\left(x_{2,1}^{b}+0\right)+B_{n_{k}}^{-} \bar{U}_{n_{k}-1}\left(x_{2, n_{k}}^{b}-0\right)=\bar{g}_{1}^{+}+\bar{g}_{n_{k}}^{-},
\end{gathered}
$$

where $B_{k}^{-}, B_{k}^{+}, k=2, \ldots, n_{k}-1, B_{1}^{+}, B_{n_{k}}^{-}$are matrices of boundary conditions of the fourth order; $\bar{g}_{k}^{-}, \bar{g}_{k}^{+}, k=2, \ldots, n_{k}-1, \bar{g}_{1}^{+}, \bar{g}_{n_{k}}^{-}$are right-side vectors of boundary conditions.

Operators (18) and (21) can be rewritten in the following form:

$$
B=\partial_{1}^{2} B_{2,0}+\partial_{1} \partial_{2} B_{1,1}+\partial_{2}^{2} B_{0,2} ; \quad B^{*}=\partial_{1}^{2} B_{2,0}^{*}+\partial_{1} \partial_{2} B_{1,1}^{*}+\partial_{2}^{2} B_{0,2}^{*},
$$

where

$$
\begin{array}{ll}
B_{2,0}=-\left[\begin{array}{llll}
1 & 0 & 0 & 0
\end{array}\right]^{T} ; B_{1,1}=-\left[\begin{array}{llll}
0 & 0 & 2 & 2
\end{array}\right]^{T} ; \quad B_{0,2}=-\left[\begin{array}{llll}
0 & 1 & 0 & 0
\end{array}\right]^{T} ; \\
B_{2,0}^{*}=-\left[\begin{array}{llll}
1 & 0 & 0 & 0
\end{array}\right] ; \quad B_{1,1}^{*}=-\left[\begin{array}{llll}
0 & 0 & 2 & 2
\end{array}\right] ; \quad B_{0,2}^{*}=-\left[\begin{array}{llll}
0 & 1 & 0 & 0
\end{array}\right] .
\end{array}
$$

Thus, we have:

$$
\begin{aligned}
\left(B^{*} \bar{A} B w, w\right) & =\left(\left(\partial_{1}^{2} B_{2,0}^{*}+\partial_{2}^{*} \partial_{1}^{*} B_{1,1}^{*}+\partial_{2}^{2} B_{0,2}^{*}\right) \bar{A}\left(\partial_{1}^{2} B_{2,0}+\partial_{1} \partial_{2} B_{1,1}+\partial_{2}^{2} B_{0,2}\right) w, w\right)= \\
& =\left(\partial_{1}^{2} B_{2,0}^{*} \bar{A} \partial_{1}^{2} B_{2,0} w, w\right)+\left(\partial_{1}^{2} B_{2,0}^{*} \bar{A} \partial_{1} \partial_{2} B_{1,1} w, w\right)+ \\
& +\left(\partial_{1}^{2} B_{2,0}^{*} \bar{A} \partial_{2}^{2} B_{0,2} w, w\right)+\left(\partial_{2}^{*} \partial_{1}^{*} B_{1,1}^{*} \bar{A} \partial_{1}^{2} B_{2,0} w, w\right)+ \\
& +\left(\partial_{2}^{*} \partial_{1}^{*} B_{1,1}^{*} \bar{A} \partial_{1} \partial_{2} B_{1,1} w, w\right)+\left(\partial_{2}^{*} \partial_{1}^{*} B_{1,1}^{*} \bar{A} \partial_{2}^{2} B_{0,2} w, w\right)+ \\
& +\left(\partial_{2}^{2} B_{0,2}^{*} \bar{A} \partial_{1}^{2} B_{2,0} w, w\right)+\left(\partial_{2}^{2} B_{0,2}^{*} \bar{A} \partial_{1} \partial_{2} B_{1,1} w, w\right)+ \\
& +\left(\partial_{2}^{2} B_{0,2}^{*} \bar{A} \partial_{2}^{2} B_{0,2} w, w\right)=\left(B_{0,2}^{*} \bar{A} B_{0,2} y_{3}, y_{3}\right)+ \\
& +\left(B_{0,2}^{*} \bar{A} \partial_{1} B_{1,1} y_{2}, y_{3}\right)+\left(\partial_{1}^{*} B_{1,1}^{*} \bar{A} B_{0,2} y_{3}, y_{2}\right)+ \\
& +\left(B_{0,2}^{*} \bar{A} B_{2,0} \partial_{1}^{2} y_{1}, y_{3}\right)+\left(\partial_{1}^{2} B_{2,0}^{*} \bar{A} B_{0,2} y_{3}, y_{1}\right)+ \\
& +\left(\partial_{1}^{*} B_{1,1}^{*} \bar{A} B_{2,0} \partial_{1}^{2} y_{1}, y_{2}\right)+\left(\partial_{1}^{2} B_{2,0}^{*} \bar{A} \partial_{1} B_{1,1} y_{2}, y_{1}\right)+ \\
& +\left(\partial_{1}^{*} B_{1,1}^{*} \bar{A} B_{1,1} \partial_{1} y_{2}, y_{2}\right)+\left(\partial_{1}^{2} B_{2,0}^{*} \bar{A} B_{2,0} \partial_{1}^{2} y_{1}, y_{1}\right)
\end{aligned}
$$

and finally

$$
\begin{aligned}
\left(B^{*} \bar{A} B w, w\right)= & \left(B_{0,2}^{*} \bar{A} B_{0,2} y_{3}, y_{3}\right)+\left(B_{0,2}^{*} \bar{A} \widetilde{B}_{1,1} y_{2}, y_{3}\right)+\left(\widetilde{B}_{1,1}^{*} \bar{A}_{k} B_{0,2} y_{3}, y_{2}\right)+ \\
& +\left(B_{0,2}^{*} \bar{A} \widetilde{B}_{2,0} y_{1}, y_{3}\right)+\left(\widetilde{B}_{2,0}^{*} \bar{A} B_{0,2} y_{3}, y_{1}\right)+\left(\widetilde{B}_{1,1}^{*} \bar{A} \widetilde{B}_{2,0} y_{1}, y_{2}\right)+ \\
& +\left(\widetilde{B}_{2,0}^{*} \bar{A} \widetilde{B}_{1,1} y_{2}, y_{1}\right)+\left(\widetilde{B}_{1,1}^{*} \bar{A} \widetilde{B}_{1,1} y_{2}, y_{2}\right)+\left(\widetilde{B}_{2,0}^{*} \bar{A} \widetilde{B}_{2,0} y_{1}, y_{1}\right),
\end{aligned}
$$


where

$$
\begin{gathered}
B_{2,0}=-\left[\begin{array}{llll}
\partial_{1}^{2} & 0 & 0 & 0
\end{array}\right]^{T} ; B_{1,1}=-\left[\begin{array}{llll}
0 & 0 & 2 \partial_{1} & 2 \partial_{1}
\end{array}\right]^{T} ; \\
B_{2,0}^{*}=-\left[\begin{array}{llll}
\partial_{1}^{2} & 0 & 0 & 0
\end{array}\right] ; \quad B_{1,1}^{*}=-\left[\begin{array}{llll}
0 & 0 & 2 \partial_{1}^{*} & 2 \partial_{1}^{*}
\end{array}\right] .
\end{gathered}
$$

We have

$$
\left(B^{*} \bar{A} B w, w\right)=\left(K \bar{U}^{r}, \bar{U}^{r}\right)
$$

where

$$
K=\left[\begin{array}{ccc}
\widetilde{B}_{2,0}^{*} \bar{A} \widetilde{B}_{2,0} & \widetilde{B}_{2,0}^{*} \bar{A} \widetilde{B}_{1,1} & \widetilde{B}_{2,0}^{*} \bar{A} B_{0,2} \\
\widetilde{B}_{1,1}^{*} \bar{A} \widetilde{B}_{2,0} & \widetilde{B}_{1,1}^{*} \bar{A} \widetilde{B}_{1,1} & \widetilde{B}_{1,1}^{*} \bar{A}_{k} B_{0,2} \\
B_{0,2}^{*} \bar{A} \widetilde{B}_{2,0} & B_{0,2}^{*} \bar{A} \widetilde{B}_{1,1} & B_{0,2}^{*} \bar{A} B_{0,2}
\end{array}\right] ; \quad \bar{U}^{r}=\left[\begin{array}{l}
y_{1} \\
y_{2} \\
y_{3}
\end{array}\right] .
$$

Thus, functional (19) can be rewritten in the following form:

$$
\Phi(w)=\Phi\left(\bar{U}^{r}\right)=\frac{1}{2} \iint_{\omega}\left(\widetilde{K} \bar{U}^{r}, \bar{U}^{r}\right) d x_{1} d x_{2}-\iint_{\omega}\left(\overline{\mathrm{F}}, \bar{U}^{r}\right) w d x_{1} d x_{2},
$$

where

$$
\widetilde{K}=\left[\begin{array}{ccc}
\widetilde{B}_{2,0}^{*} \bar{A} \widetilde{B}_{2,0}+\bar{c} & \widetilde{B}_{2,0}^{*} \bar{A} \widetilde{B}_{1,1} & \widetilde{B}_{2,0}^{*} \bar{A} B_{0,2} \\
\widetilde{B}_{1,1}^{*} \bar{A} \widetilde{B}_{2,0} & \widetilde{B}_{1,1}^{*} \bar{A} \widetilde{B}_{1,1} & \widetilde{B}_{1,1}^{*} \bar{A}_{k} B_{0,2} \\
B_{0,2}^{*} \bar{A} \widetilde{B}_{2,0} & B_{0,2}^{*} \bar{A} \widetilde{B}_{1,1} & B_{0,2}^{*} \bar{A} B_{0,2}
\end{array}\right] ; \quad \overline{\mathrm{F}}=\left[\begin{array}{c}
\mathrm{F} \\
0 \\
0
\end{array}\right]
$$

The solution of this problem is the point (function) of the constrained extremum of this functional with the condition (27), taking into account (32), (33).

These formulations are the initial ones for the realization of the discrete-continual methods for the problems of anisotropic plate analysis.

The Reported study was Funded by Government Program of the Russian Federation "Development of science and technology" (2013-2020) within Program of Fundamental Researches of Ministry of Construction, Housing and Utilities of the Russian Federation and Russian Academy of Architecture and Construction Sciences, the Research Project 7.1.1".

\section{References}

1. P.A. Akimov, Applied Mechanics and Materials, 204-208, 4502-4505 (2012).

2. P.A. Akimov, M.L. Mozgaleva, Applied Mechanics and Materials, 580-583, 28982902 (2014).

3. K.-J. Bathe, Finite Element Procedures (Prentice Hall Inc., 1996).

4. O.C. Zienkiewicz, R.L. Taylor, D.D. Fox, The Finite Element Method for Solid and Structural Mechanics (Butterworth-Heinemann, 2013).

5. O.C. Zienkiewicz, R.L. Taylor, J.Z. Zhu, The Finite Element Method: Its Basis and Fundamentals (Butterworth-Heinemann, 2005).

6. A.B. Zolotov, P.A. Akimov, Proceedings of the International Symposium LSCE 2002 organized by Polish Chapter of IASS (Warsaw, Poland, 2002). 